\title{
Primary Stroke Centers: Their Role and Impact on Acute Stroke Management
}

\author{
Carissa C. Pineda MD \\ Thomas Jefferson University \\ Jeanne Birch RN, BSN \\ Thomas Jefferson University
}

Follow this and additional works at: https://jdc.jefferson.edu/jhnj

Part of the Neurology Commons

Let us know how access to this document benefits you

\section{Recommended Citation}

Pineda MD, Carissa C. and Birch RN, BSN, Jeanne (2008) "Primary Stroke Centers: Their Role and Impact on Acute Stroke Management," JHN Journal: Vol. 4 : Iss. 1 , Article 4.

DOI: https://doi.org/10.29046/JHNJ.004.1.001

Available at: https://jdc.jefferson.edu/jhnj/vol4/iss1/4

This Article is brought to you for free and open access by the Jefferson Digital Commons. The Jefferson Digital Commons is a service of Thomas Jefferson University's Center for Teaching and Learning (CTL). The Commons is a showcase for Jefferson books and journals, peer-reviewed scholarly publications, unique historical collections from the University archives, and teaching tools. The Jefferson Digital Commons allows researchers and interested readers anywhere in the world to learn about and keep up to date with Jefferson scholarship. This article has been accepted for inclusion in JHN Journal by an authorized administrator of the Jefferson Digital Commons. For more information, please contact: JeffersonDigitalCommons@jefferson.edu. 


\section{Primary Stroke Centers: Their Role and Impact on Acute Stroke Management}

\author{
Carissa C. Pineda MD' and and Jeannee Birch RN, BSN² \\ Department of ${ }^{1}$ Neurology and ${ }^{2}$ Department of Performance Improvement, \\ Thomas Jefferson University, Philadelphia, Pennsylvania
}

The management of acute ischemic stroke has been recognized as a significant medical problem. Stroke remains to be the third leading cause of death and the leading cause of long-term disability; $80 \%$ of all strokes are ischemic (a blood clot disrupts blood flow), and the rest are hemorrhagic (a blood vessel ruptures in the brain). Nearly 1 in 15 deaths in 2003 were the result of a stroke. ${ }^{1}$ Approximately 750,000 new strokes occur annually, of which 250,000 result in the deaths in the United States alone. The latest estimates for stroke costs total to about $\$ 30$ billion in direct costs, and $\$ 20$ billion are in indirect costs. ${ }^{2}$ If nothing is done about this disease, the annual incidence of strokes is expected to reach 1.1 million by the year 2015 .

Despite recent advances in its diagnosis and treatment, a population of the general public still have difficulty recognizing stroke symptoms, and are unaware that stroke is indeed a medical emergency. ${ }^{3}$ The two major causes of delay in treatment are mainly failure to appropriately recognize the symptoms that indicate stroke (numbness, dizziness, confusion, or headache), and failure to access a medical facility that is equipped to handle acute strokes. In an effort to raise awareness, and the level of care provided to stroke patients, stroke center standards were created and hospitals that met the strict criteria were then selected. However, many hospitals do not have the necessary infrastructure and organization needed to triage and treat patients with stroke rapidly and efficiently. ${ }^{4}$

Seeing that the model of trauma centers were effective both in the rural and urban settings, the concept of acute stroke centers were then explored. The Brain Attack Coalition, which is a multidisciplinary group involved in stroke care, set out to establish guidelines for stroke centers. The role of a Primary Stroke Center is to be able to stabilize and provide emergency care for the acute stroke patients. Depending on the complexity of the case, they would either admit the patient, or transfer them to a comprehensive stroke center. On the other hand, the Comprehensive Stroke Center should be able to provide complete care to any complex patient; they should have the necessary infrastructure, both in personnel and equipment.

The main goals and benefits of a certified stroke center are the following:

- Improve the efficiency of patient care

- Provide access to the most up to date stroke therapies, which ultimately will lead to fewer peristroke complications

- Increase the number of patients discharged home than to skilled nursing facilities

- Decrease the length of hospital stay.

By accomplishing all of the above, the cost to the healthcare system will be reduced, and hopefully, patient satisfaction should also increase.

Although advances in stroke treatment have moved forward over the past years, the main limitation remains to be the prompt and proper treatment. Thrombolytic therapy, which has been FDA approved for over 10 years now remains to be the mainstay of acute treatment, however, only about $3 \%$ of patients with acute strokes are being treated. Another important goal of stroke centers is to provide access to these acute therapies.

The Brain Attack Coalition and American Stroke association have developed a framework of requirements needed to establish a Primary Stroke Center. These elements are based on the use of standardized method of delivering evidence based clinical care with known improvement of patient outcome. The Joint Commission has recognized the effectiveness of these measures and has based their criteria for Primary Stroke Center Certification on the recommendations listed in the accompanying table. ${ }^{6-8}$

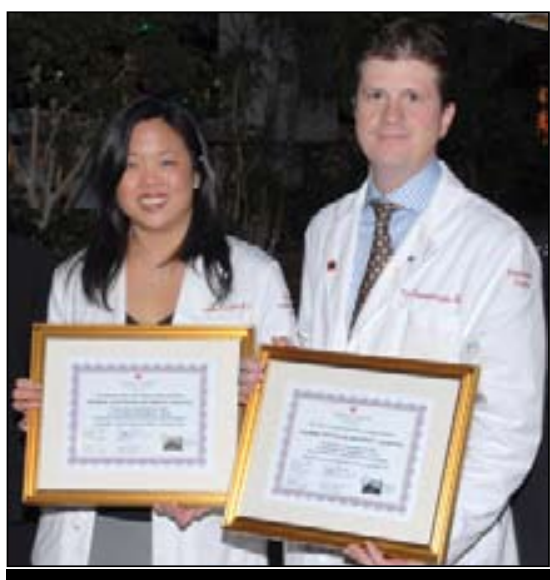

Drs. Erol Veznedaroglu

(Neurosurgery) and Carissa Pineda (Neurology) with the Gold and Silver Performance Awards from the American Heart Association for maintained high standards in care and management of stroke patients.

In this decade, the integration of multidisciplinary approach in the treatment of strokes will occur within the structure of a stroke center. Establishing a Stroke Center requires coordination of efforts throughout the Health System. Even hospitals with limited resources can positively impact the outcome of a stroke patient. Smaller healthcare centers can develop a "Stroke Network" with larger healthcare centers to provide comprehensive care. The most crucial component of stroke care is time- "Lost time equals lost brain" is the slogan, as brain damage progresses the blood flow is cut off. Rapid evaluation and referral with "Treat and Transfer" protocols can be established at any hospital that has the ability to provide Emergency Services. With new therapies emerging for stroke treatment, this could significantly impact all patients having access to cutting edge stroke care.

The American Heart Association's Performance Awards for hospitals is based on $85 \%$ compliance for patients in each of the following 
items. The Silver Award is given to institutions who meet goals for 12 months and the Gold Award, for 24 months.

- Percent of acute ischemic stroke patients who arrive at the ED within 120 minutes (two hours) of onset of stroke symptoms who receive IV t-PA within 180 minutes (three hours) of onset of stroke symptoms

- Percent of ischemic stroke or TIA patients who receive antithrombotic medication within 48 hours of hospitalization
- Percent of ischemic stroke or TIA patients discharged on antithrombotics (e.g., warfarin, aspirin, other antiplatelet drug)

- Percent of ischemic stroke or TIA patients with atrial fibrillation who are discharged on anticoagulation therapy (warfarin/Coumadin or heparin/heparinoids) unless an absolute or relative contraindication exists

- Percent of patients at risk for DVT who received DVT prophylaxis by the second hospital day

\section{Acute Stroke Team}

This is composed of physicians and health care professionals with experience and proper training in management of acute strokes. They should be available $24 \mathrm{hrs}$ a day to evaluate patients with symptoms suggestive of an acute stroke. The institution should have a Stroke notification system in place with documented expected response times.

\section{Written Care Protocols}

One of the major key elements in developing good outcomes is through the implementation of protocols. As such, protocols should be based on well established Standards of Care and national guidelines. This should include emergency care of ischemic/hemorrhagic stroke patients. Studies have shown that by adhering to protocol guidelines, more patients were properly screened for tPA. Not only was there a substantial increase in the proportion of patients receiving thrombolytic therapy, but rather, there was also a decrease in the rates of symptomatic ICH. ${ }^{5}$ If the patient is deemed to be complex, a protocol should be in place for those requiring transfers to a comprehensive center-a "Treat and Transfer" pathway should be initiated.

\section{Emergency Department}

The ED serves as the gateway between the patient and the hospital. It is important that emergency personnel are trained in recognizing, diagnosing, and treating all types of acute stroke. There should be highly organized means communication amongst the EMS, ED personnel and the Acute Stroke Team. The ED should be able to provide proper documentation of patient's history, procedures, and qualifications for thrombolytic therapy.

\section{Stroke Unit}

This is a dedicated unit staffed by health care personnel (vascular neurologists, stroke-trained nurses, physical/occupational/speech therapists, case managers, social workers) who are trained to care for patients with cerebrovascular diseases. This unit should also be equipped with infrastructure such as continuous telemetry, and the capability for blood pressure monitoring. There has been a big difference noted in the care received by stroke patients in the stroke unit setting as compared to the general medical floors. Patients in stroke units had an $8 \%$ reduction in length of stay, and $7 \%$ increase in going back to living at home, and a $17 \%$ reduction in death rates. The cost of forming and operating stroke units will vary depending on size and location. Again, there is a need for written care protocols for standardization of care.

\section{Neurosurgery}

During the acute phase of a stroke, patients may need a neurosurgical procedure, thus, a primary stroke center must be able to provide neurosurgical care within 2 hours if clinically necessary. If this is not met, the patient should be transferred to a medical facility with an available neurosurgeon who is experienced in treating different types of acute strokes. A protocol is necessary to Treat and Transfer patients with the need for comprehensive stroke care.

- Percent of ischemic stroke or TIA patients with $\mathrm{LDL}>=100 \mathrm{mg} / \mathrm{dL}$ OR on cholesterol reducer prior to admission who are discharged on cholesterol reducing drugs

- Percent of smokers who receive smoking cessation advice or medication (e.g., Nicoderm or Zyban) at discharge

These steps are all based on published evidence assessing modifiable risk factors which affect the likelihood of stroke. ${ }^{9,} 10$ Any one of these risk factors can double the chance of a stroke recurring.

\section{Neuroimaging}

In order to have a proper stroke diagnosis, it is vital for a primary stroke center to have imaging capability 24 hours a day. The ideal door to imaging time is within 25 minutes of order entry, and must be interpreted within 20 minutes.

\section{Hospital and Administrative Support}

There should be a designated Medical Director of Stroke Center with expertise and training in Cerebovascular Diseases. Commitment from administration of hospital/health system is important in providing support and funding for the needed resources to maintain the ability to deliver high quality and efficient care to acute stroke patients and the community.

\section{Laboratory Services}

This should be available $24 \mathrm{hrs}$. a day (CBC, Chemistry and Coagulation studies) The chest $\mathrm{x}$-ray and EKG must be completed within 45 minutes of patient's arrival

\section{Outcome/Performance Improvement:}

PrimaryStroke Centers must be able to develop specific stroke performance measures to be monitored by Performance Improvement Department, there should be ongoing efforts with Stroke Team and Performance Improvement to improve stroke care by utilizing benchmarking and performance measures data

\section{Educational Programs}

In order to keep up with new developments in stroke care, the stroke team professional staff is required to maintain 8 hours CME (or its equivalent) credits per year. In addition to this, the stroke center should be able to offer education to the community at least twice a year in relation to Stroke recognition, prevention, and treatment. 


\section{References}

1. American Heart Association. Heart Disease and Stroke Statistics-2006 Update; American Heart Association; 2006.

2. Stroke Treatment and Ongoing Prevention Act of 2001. S. 1274/H.R. 3431 (2001)

3. Dion, J. Management of Ischemic Stroke in the Next Decade: Stroke Centers of Excellence. Journal of Vasc. Interventional Radiology 2004; 15:S133-S141

4. Alberts, M. et al. Recommendations for the Establishment of Primary Stroke Centers. JAMA, 2000; 283:3102-3109
5. Katzan et al. Quality Improvement and Tissue-Type Plasminogen Activator for Acute Ischemic Stroke: A Cleveland Update Stroke, 2003; 34:799-800

6. Adams, R, Acker, J et. Al. Recommendations for Improving the Quality of Care Through Stroke Centers and Systems: An Examination of Stroke Center Identification Options. Stroke, 2002; 33:e1-e7

7. Schwamm, L.H., Pancioli, A, et al. Recommendations for the Establishment of Stroke Systems of Care,

Recommendations from the American Stroke Association's Task Force on the Development of Stroke Systems., Stroke 2005;36:690-703
8. The National Institute of Neurological Disorders and Stroke (NINDS) rt-PA Stroke Study Group; A Systems Approach to Immediate Evaluation and Management of Hyperacute Stroke : Experience at Eight Centers and Implications for Community Practice and Patient Care. Stroke 1997;28:1530-1540

9. Sacco, R. L., Adams, R., et al. Guidelines for Prevention of Stroke in Patients With Ischemic Stroke or Transient Ischemic Attack. Stroke 2006;37:577-617

10. Goldstein, L. B., Adams, R., et al. Primary Prevention of Ischemic Stroke. Stroke 2006;37:1583-1633 